\title{
Prefrontal Cortical GABA Transmission Modulates Discrimination and Latent Inhibition of Conditioned Fear: Relevance for Schizophrenia
}

\author{
Patrick T Piantadosi' and Stan B Floresco*,' \\ 'Department of Psychology and Brain Research Centre, University of British Columbia, Vancouver British Columbia, Canada
}

\begin{abstract}
Inhibitory gamma-aminobutyric acid (GABA) transmission within the prefrontal cortex (PFC) regulates numerous functions, and perturbations in GABAergic transmission within this region have been proposed to contribute to some of the cognitive and behavioral abnormalities associated with disorders such as schizophrenia. These abnormalities include deficits in emotional regulation and aberrant attributions of affective salience. Yet, how PFC GABA regulates these types of emotional processes are unclear. To address this issue, we investigated the contribution of PFC GABA transmission to different aspects of Pavlovian emotional learning in rats using translational discriminative fear conditioning and latent inhibition (LI) assays. Reducing prelimbic PFC GABA $A_{A}$ transmission via infusions of the antagonist bicuculline before the acquisition or expression of fear conditioning eliminated the ability to discriminate between an aversive conditioned stimulus (CS + ) paired with footshock vs a neutral CS-, resembling similar deficits observed in schizophrenic patients. In a separate experiment, blockade of PFC GABA receptors before CS preexposure (PE) and conditioning did not affect subsequent expression of LI, but did enhance fear in rats that were not preexposed to the CS. In contrast, PFC GABA-blockade before a fear expression test disrupted the recall of learned irrelevance and abolished LI. These data suggest that normal PFC GABA transmission is critical for regulating and mitigating multiple aspects of aversive learning, including discrimination between fear vs safety signals and recall of information about the irrelevance of stimuli. Furthermore, they suggest that similar deficits in emotional regulation observed in schizophrenia may be driven in part by deficient PFC GABA activity.

Neuropsychopharmacology (2014) 39, 2473-2484; doi:I0.I038/npp.20I4.99; published online 28 May 20I4
\end{abstract}

\section{INTRODUCTION}

Maladaptive assignment of affective salience to environmental stimuli has been hypothesized to underlie many of the behavioral pathologies characteristic of schizophrenia (Jensen et al, 2008; Kapur, 2003; Palaniyappan and Liddle, 2012). For example, delusional ideation may be supported by aberrant associative learning in relation to otherwise innocuous stimuli (Kapur, 2003; Palaniyappan and Liddle, 2012). Similarly, the failure to apply appropriate affective importance to hedonic or aversive stimuli may result in negative symptoms, including anhedonia and avolition (Orliac et al, 2013; Walter et al, 2010). Deficient filtering of task-irrelevant stimuli may also impair cognitive functioning (Anticevic et al, 2011).

Schizophrenia is associated with abnormal modulation of activity by emotionally relevant stimuli in numerous corticolimbic brain regions (Murray et al, 2008; Roiser

\footnotetext{
*Correspondence: Dr SB Floresco, Department of Psychology and Brain Research Center, University of British Columbia, 2136 West Mall, Vancouver, BC, Canada V6T IZ4, Tel: + 604827 5313, Fax: + 604822 6923, E-mail: floresco@psych.ubc.ca Received 5 March 20I4; revised II April 2014; accepted 28 April 20।4; accepted article preview online 2 May 2014
}

et al, 2013; Taylor et al, 2005). In particular, the prefrontal cortex (PFC) encodes affective salience in humans (Roiser et al, 2010) and rodents (Moessnang et al, 2012), and is thought to be an area of primary pathology in schizophrenia (Lewis et al, 2004; Moghaddam, 2002; Volk and Lewis, 2002). For example, functional imaging studies have revealed that activation of the medial PFC (MPFC) is greater in response to a neutral conditioned stimulus (CS-) than an appetitive CS (CS +), a pattern opposite of that observed in controls (Diaconescu et al, 2011). Similar patterns of maladaptive PFC activation in response to neutral stimuli have been observed using an aversive conditioning paradigm, suggesting a neural correlate independent of the specific hedonic nature of the stimuli used (Jensen et al, 2008).

Although these studies point to prefrontal dysfunction as a contributing factor to maladaptive affective processing, less is known about the neurochemical basis for these deficits. It has been hypothesized that schizophrenia is associated with a shift in the balance of cortical excitatory/ inhibitory transmission, leading to disorganized excitation through alterations in excitatory glutamate and inhibitory gamma-aminobutyric acid (GABA) transmission (GonzalezBurgos and Lewis, 2012; Moghaddam and Javitt, 2011; Moghaddam and Krystal, 2012; O'Donnell, 2011). This may be driven in part by deficiencies in GABAergic transmission 
within the frontal lobes, as reductions in markers for GABA interneurons in post-mortem PFC of individuals with schizophrenia are some of the more reliable cellular abnormalities associated with the disorder (Akbarian et al, 1995; Benes and Berretta, 2001; Gonzalez-Burgos et al, 2010; Lewis et al, 2012). Dysfunction in PFC GABA transmission has been hypothesized to contribute to cognitive deficits observed in schizophrenia. As such, preclinical investigations probing how PFC GABA transmission regulates various cognitive and emotional functions can provide important insight into how dysfunction in this system may contribute to schizophrenia symptomology.

Recent studies have utilized intra-mPFC microinfusion of $\mathrm{GABA}_{\mathrm{A}}$-receptor antagonists to mimic the disinhibition thought to result from endemic alterations in cortical excitatory/inhibitory balance and assess how this may alter behaviors relevant to schizophrenia. Reducing mPFC $\mathrm{GABA}_{\mathrm{A}}$ transmission in rats produces cognitive and neurophysiological alterations reminiscent of schizophrenia, including attentional deficits (Asinof and Paine, 2013; Paine et al, 2011; Pehrson et al, 2013), impaired set-shifting, speed-of-processing during working memory, and increases in behavioral and neurophysiological indices of dopamine function (Enomoto et al, 2011). Thus, these manipulations may serve as a useful tool to assess the impact of cortical GABAergic hypofunction on other process that are perturbed in schizophrenia, such as affect regulation.

Reverse-translational assessment of affective conditioning can be conducted using Pavlovian discriminative fear conditioning and latent inhibition (LI) assays in rats. LI has been reported to be deficient in acutely psychotic individuals (Gray et al, 1995; Rascle et al, 2001) and is thought to represent the inability to appropriately filter irrelevant stimuli, representing aberrant affective salience attribution (Gray and Snowden, 2005). Similarly, the ability to distinguish between aversive and neutral conditioned stimuli is markedly disturbed in schizophrenia (Jensen et al, 2008) and can be assessed in rodents using a discriminative fear conditioning task. In this study, we evaluated the importance of $\mathrm{GABA}_{\mathrm{A}}$ transmission in the mPFC of rats on the adaptive assignment of salience to conditioned environmental stimuli utilizing these two assays, to provide insight into how abnormalities in affective processes may be related to reduced PFC GABA transmission.

\section{MATERIALS AND METHODS}

\section{Subjects and Surgery}

Cohorts of male Long Evans rats (Charles River Laboratories, Montreal, QC) weighing 250-300 g at the start of the experiment were initially group housed and provided with ad libitum access to food and water. Following a week of acclimatization to the colony, animals were stereotaxically implanted with bilateral guide cannula into the prelimbic region of $\mathrm{MPFC}$, described below. For the remainder of the experiment, rats were singly housed and food restricted to approximately $90 \%$ of their free feeding weight. Each experiment was conducted in separate cohorts of approximately 16 rats. Care was taken to ensure that, for each cohort, comparable numbers of rats were assigned to each experimental condition to avoid potential cohort affects. All testing was conducted in accordance with Canadian Council on Animal Care and the Animals Care Committee of the University of British Columbia.

Rats were anesthetized with a combination of ketamine/ xylazine $(100 / 10 \mathrm{mg} / \mathrm{kg}$ i.p.) and supplemented with analgesia (Anafen, $10 \mathrm{mg} / \mathrm{kg}$ s.c.). Twenty-three gauge bilateral stainless-steel guide cannula were aimed at the prelimbic $\mathrm{mPFC}$ according to the following coordinates (Paxinos and Watson, 2005) from bregma: AP: $+3.2 \mathrm{~mm}$; ML: $\pm 0.7 \mathrm{~mm}$; from dura: DV: -2.8 , with the intraural bar set to $-3.3 \mathrm{~mm}$. Dental acrylic adhered to four stainless-steel skull screws held cannula in place. Stainless steel obdurators flush with the end of the guide cannula were inserted after surgery. Rats were given approximately 1 week to recover from surgery before beginning behavioral training.

\section{Apparatus}

Behavioral testing was conducted in eight standard operant chambers $(30.5 \times 24 \times 21 \mathrm{~cm}$; Med Associates, St Albans, VT). Chambers were housed in a sound attenuating enclosure equipped with a fan providing ventilation and masking ambient noise. Each chamber was fitted with two retractable levers along one wall, separated by a food receptacle where sucrose reinforcement was delivered (45 mg pellet; BioServ, Frenchtown, NJ). For these experiments, only the left lever was extended into the chamber. Two $100 \mathrm{~mA}$ cue lights resided above each lever. On the wall opposite the food receptacle, a single $100 \mathrm{~mA}$ house light was situated directly next to an auditory speaker, which allowed for the delivery of auditory stimuli via a programmable generator (ANL-926, Med Associates). Four infrared photobeams located just above the grid floors were used to measure locomotor activity. The floor of each chamber consisted of 19 stainless steel rods spaced $1.5 \mathrm{~cm}$ apart. The rods were wired to a shock source and solid-state grid scrambler for the delivery of footshock.

\section{Microinfusion}

To acclimatize animals to the microinfusion procedure, rats received 1-2 days of mock infusions 10 min before the final lever pressing session before aversive conditioning. These consisted of obdurator removal, insertion of a mock injector flush with the end of the guide cannula, and placement in the infusion enclosure for approximately $2 \mathrm{~min}$. All microinfusions were conducted $10 \mathrm{~min}$ before animals being placed in operant chambers. On the infusion day, microinjectors extending $0.8 \mathrm{~mm}$ beyond the guide cannula were lowered into the brain and animals received bilateral infusion of $0.9 \%$ saline $(0.5 \mu \mathrm{l} / \mathrm{side})$ or bicuculline methobromide in a $0.9 \%$ saline solution $(50 \mathrm{ng} / \mu \mathrm{l}$ at a volume of $0.5 \mu 1 /$ side). Previous studies have shown that this dose of bicuculline is effective at inducing cognitive and neurophysiological alterations, whereas lower doses (12.5 ng) are less effective (Enomoto et al, 2011; Paine et al, 2011). Moreover, this dose is orders of magnitudes lower than those used to induce epileptiform activity (Schneider and de Lores Arnaiz, 2013). Infusion was conducted over $75 \mathrm{~s}$, with injectors left in place for $60 \mathrm{~s}$ to allow for diffusion from cannula tips. 


\section{Lever Press Training}

For all experiments, rats were initially trained to press the left lever for sucrose pellet reward. Twenty-four hours before their first operant training session, rats were provided with $\sim 30$ pellets in their home cage. Training sessions were conducted at a consistent time each day. Over 1-3 days, rats learned to press using a fixed ratio 1 (FR1) schedule of reinforcement, at a criterion of 40 total presses in $30 \mathrm{~min}$. Over the next 3 days, animals were trained on an increasing variable interval (VI) schedule whereby reward was provided every 15 (VI15), 30 (VI30), or 60 (VI60) s (one session at each schedule, per day). Lever pressing on a VI60 ratio engenders a high rate of lever-press responding in rats, although the amount of reward received is relatively independent of response rates, which allows for accurate assessment of conditioned suppression as a proxy for fear (McAllister, 1997; Quirk et al, 2000). Training with the VI60 schedule continued for 9-11 days, after which aversive conditioning commenced.

\section{Discriminative Fear Conditioning}

Conditioning. Rats underwent discriminative fear conditioning modeled after the protocol described by Antunes and Moita (2010) (see Figure 1a), which is similar to one used in human subjects (Balog et al, 2013; Jensen et al, 2008). In this protocol, rats received a total of eight presentations each of a neutral CS (CS-) and an aversive CS $(\mathrm{CS}+)$. Animals were placed in the chambers (no levers or house light) and initially received two presentations of a $30 \mathrm{~s}$ CS- (1 kHz, $80 \mathrm{~dB}$ tone + illumination of the cue lights). Subsequently, animals were pseudorandomly presented with six more CS-, and seven 30-s presentations of the $\mathrm{CS}+(9 \mathrm{kHz}, 80 \mathrm{~dB}$ tone + flashing houselight co-terminating with $0.5 \mathrm{~mA} / 1 \mathrm{~s}$ footshock). The session ended following one additional $\mathrm{CS}+$ presentation (ie, eight presentations each of the CS- and CS + in total). The average interstimulus interval was $3 \mathrm{~min}$. The particular tones associated with $\mathrm{CS}+/ \mathrm{CS}$ - were selected because rats trained in a discriminative fear conditioning protocol tend to generalize their fear responses toward a $22 \mathrm{kHz}$ tone, which corresponds to the frequency of alarm calls (Bang et al, 2008). Thus, the lower frequency $(1 \mathrm{kHz})$ was used as the CS- to avoid biasing results toward generalization (lack of discrimination), which may arise from auditions similar to alarm calls, triggering innate fear. In addition, the visual stimuli and order of presentation were used because pilot studies revealed that this combination of stimuli produced the most robust and reliable discriminative fear responses in control animals. The day after this conditioning session, animals were given a baseline VI60 session (no shocks or CSs).

Discriminative fear test. Forty-eight hours after conditioning ( $24 \mathrm{~h}$ after baseline VI60), rats were placed in the operant chamber and allowed to lever press for food on a VI60 schedule for $5 \mathrm{~min}$, after which the presentation of CSs commenced. As a rat lever pressed, the 30-s CS- was presented four times (5-min interstimulus interval), followed by four 30-s presentations of the CS + (no shocks; 5-min interstimulus interval). The main dependent variable was conditioned suppression of lever pressing during CS presentation, used as an index of conditioned fear. Animals naturally suppress instrumental responding during exposure to an aversive CS, lending this behavior to the assessment of conditioned fear (Kamin et al, 1963; Quirk et al, 2000; Sierra-Mercado et al, 2011). This measure was used because previous work by our group has shown that intra-PFC infusions of bicuculline can increase locomotor activity when rats are not engaged in operant responding (Enomoto et al, 2011), which could confound interpretation using other measures of fear, such as freezing. Suppression was calculated by taking $((A-B) /(A+B))$, where $A$ was the number of lever presses over the $30 \mathrm{~s}$ before the CS presentation, and $B$ was the number of lever presses during the 30-s CS presentation. Thus, a suppression ratio of 1 indicates complete suppression of lever pressing, while a ratio of 0 indicates no suppression.

We tested the effects of intra-mPFC infusions of saline or bicuculline in four groups of rats. Two groups received either intra-PFC infusions of saline or bicuculline before the conditioning phase, and were tested drug-free $48 \mathrm{~h}$ later. Another two groups received either saline or bicuculline infusions before the test phase. Rats were matched for the mean number of lever press over the last 2 days of VI60 training and then assigned to one of the four groups.

\section{Latent Inhibition}

Conditioning. The LI task (Figure 1b) used was adapted from McAllister (1997), as used by Enomoto et al (2011). Following lever press training, rats were allocated to separate groups (see below), based on the mean number of lever presses made over the previous 2 VI60 days. This experiment consisted of three primary treatment groups: (1) intra-mPFC bicuculline before conditioning, (2) bicuculline before the LI test session, and (3) saline infusions before conditioning and test, which served as the control group. Rats receiving each of these treatments were further allocated to either PE or nonprexposure (NPE) conditions during the conditioning phase. For the PE condition, rats were placed in the operant chamber (no levers/house light), and experienced 30 presentations of a 30 -s compound CS (illumination of the cue-lights $+5 \mathrm{kHz}, 90 \mathrm{~dB}$ tone). Each CS presentation was separated by $30 \mathrm{~s}$. In the NPE group, rats were placed in the chambers for the same amount of time without any exposure to the CS. Thirty-six minutes after the start of the session, all rats received three pairings of the 30 -s CS co-terminating with a $0.5 \mathrm{~mA} / 0.5 \mathrm{~s}$ footshock. The next day, animals were given a baseline VI60 session.

LI test. Forty-eight hours after conditioning ( $24 \mathrm{~h}$ after baseline VI60), rats were placed in the operant chamber and allowed to lever press for reward on a VI60 schedule. Five minutes into the session, rats received the first of four 30-s CS presentations, with an intertrial interval of $5 \mathrm{~min}$. Lever press suppression during the CS presentation again served as our index of fear, and was calculated in the same manner as for discriminative fear.

\section{Histology}

Following experimental end point, rats were killed with $\mathrm{CO}_{2}$ and brains were removed and fixed in a $4 \%$ formalin 


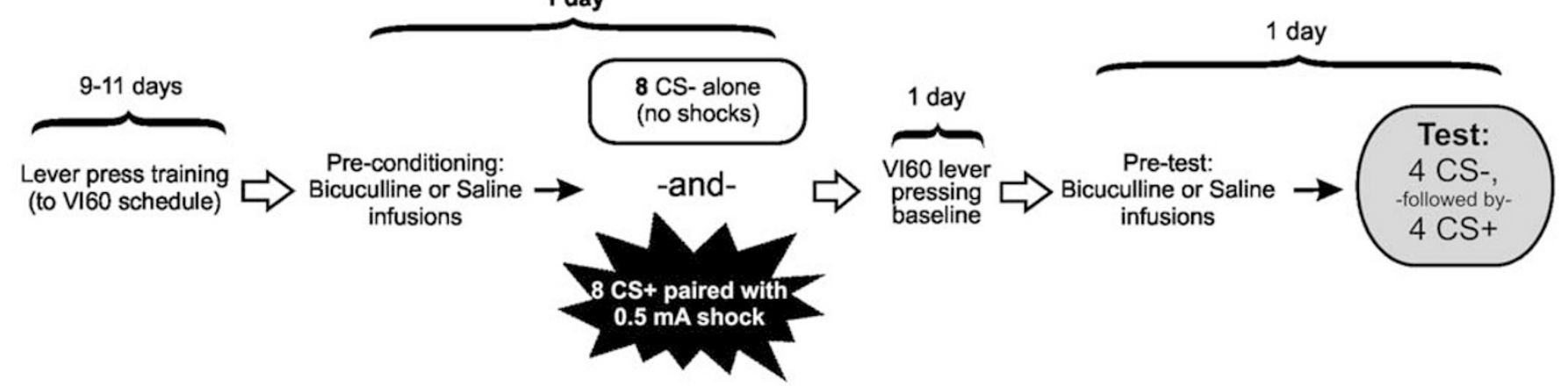

b Latent Inhibition Conditioning: 1 day
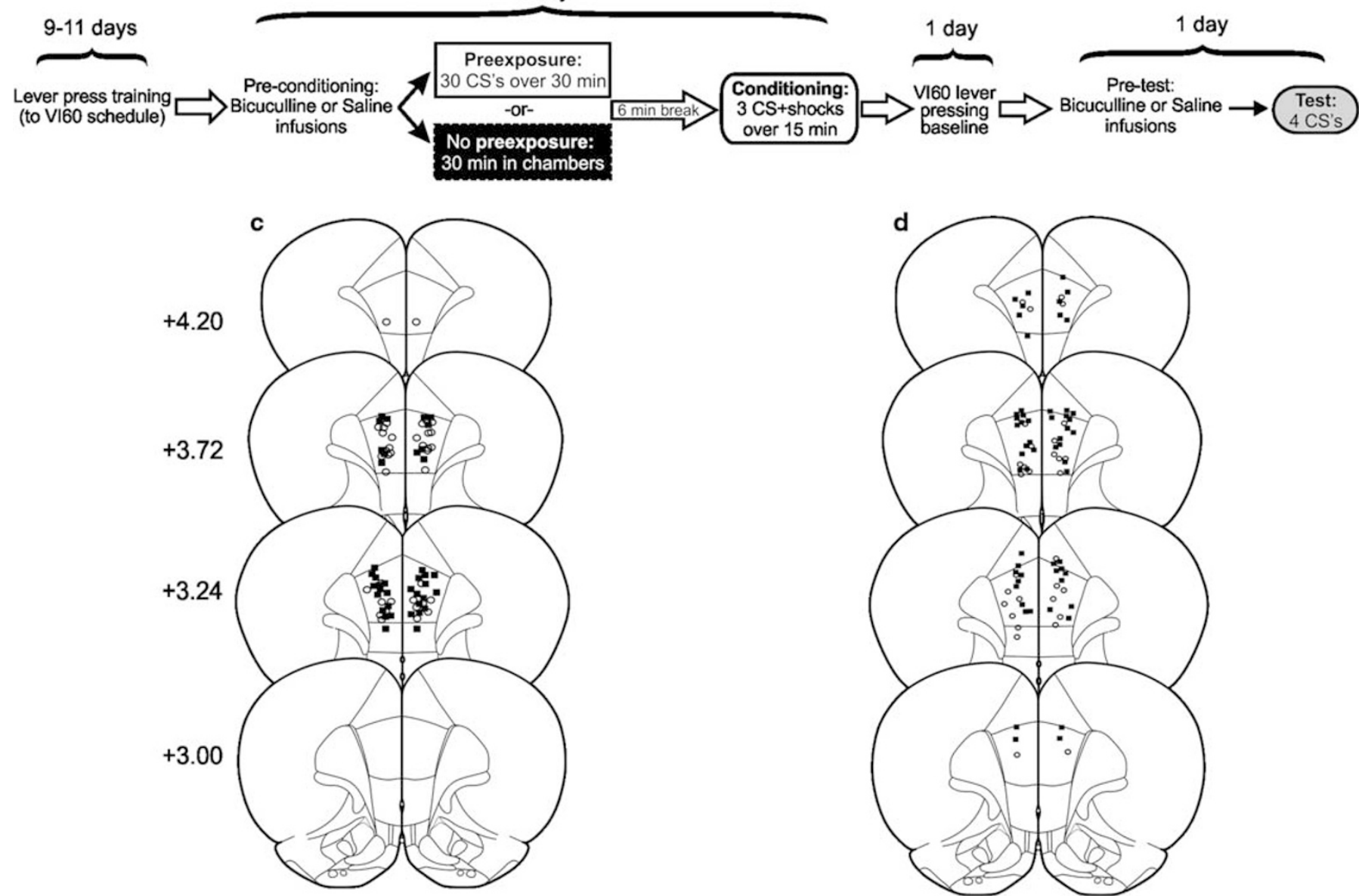

Figure I Task design and histology for ( $a, c)$ discriminative fear conditioning and (b, d) LI experiments. Note that separate groups of animals were used for saline and bicuculline infusion and for pre-conditioning and pre-test infusion. Infusion locations in the prelimbic mPFC for (c) discriminative fear conditioning and (d) LI tasks. Black squares denote animals that received bicuculline, open circles represent saline-infused animals. Distance anterior to bregma $(\mathrm{mm})$ is reflected on the left of each plate.

solution. Brains were sectioned at $50 \mu \mathrm{m}$, mounted and Nissl stained using Cresyl Violet. The ventral extent of cannula placements for the discriminative fear (Figure 1c) and LI (Figure 1d) tasks illustrate placements clustering in the prelimbic mPFC. Nine animals in the LI (six bicuculline and three saline animals) and five in the discriminative fear experiments (two bicuculline and three saline) were removed from statistical analysis because of placements exclusively outside the prelimbic mPFC.

\section{Data Analysis}

Discriminative fear conditioning was analyzed separately for pre-conditioning and pre-test infusions. Suppression ratios were analyzed using between/within-subjects 
three-way ANOVAs with Treatment group (saline vs bicuculline) as the between-subjects variable, and stimulus Type (CS + vs CS-) and stimulus Number (1-4) as the within-subjects variables. Locomotion across the entire session and the rates of lever pressing during the first $5 \mathrm{~min}$ of the session were analyzed separately using $t$-tests or ANOVAs to determine if there were any nonspecific drug effects. The rate of lever pressing during the first $5 \mathrm{~min}$ of the session were chosen for analysis as any differences between groups that emerged in the later part of the session may have been influenced by CS presentation. Follow-up simple-main effects analyses were conducted with one-way ANOVAs or $t$-tests, where appropriate.

Conditioned suppression data from the LI experiment were analyzed using two and three-way between/withinsubjects ANOVAs. LI was initially assessed using a two-way ANOVA comparing suppression during only the first CS presentation, with PE/NPE and Treatment group (bicuculline infusions before conditioning, before test or saline infusions) as between-subjects variables. To assess further changes in fear-related behaviors that were apparent during subsequent CS presentations, a three-way ANOVA was conducted with Tone as the within-subjects variable, and $\mathrm{PE} / \mathrm{NPE}$ and Treatment group again serving as between- subjects variables. Locomotion and lever pressing rates during the first $5 \mathrm{~min}$ of the session were analyzed separately using one-way ANOVAs. Follow-up simple-main effects analyses were conducted with one-way ANOVAs or $t$-tests, where appropriate.

\section{RESULTS}

\section{Discriminative Fear Conditioning}

Pre-conditioning manipulations. Control rats $(n=12)$ displayed clear discrimination between the aversive CS + and a non-aversive CS-, showing minimal suppression of lever pressing during CS- presentations and robust suppression during subsequent presentations of the shockassociated $\mathrm{CS}+$ (Figure 2a, left). In stark contrast, GABAreceptor antagonism before conditioning $(n=13)$ abolished fear discrimination, with rats displaying no discernible discrimination between each CS (Figure 2a, right). Analyses of these data revealed a significant Treatment $\times$ Stimulus type interaction, $(F(1,23)=15.76, p<0.001)$. Simple-main effects analyses confirmed that control rats displayed significantly greater levels of suppression during the CS + $v s$ the CS- $(p<0.001)$, but there was no difference on this
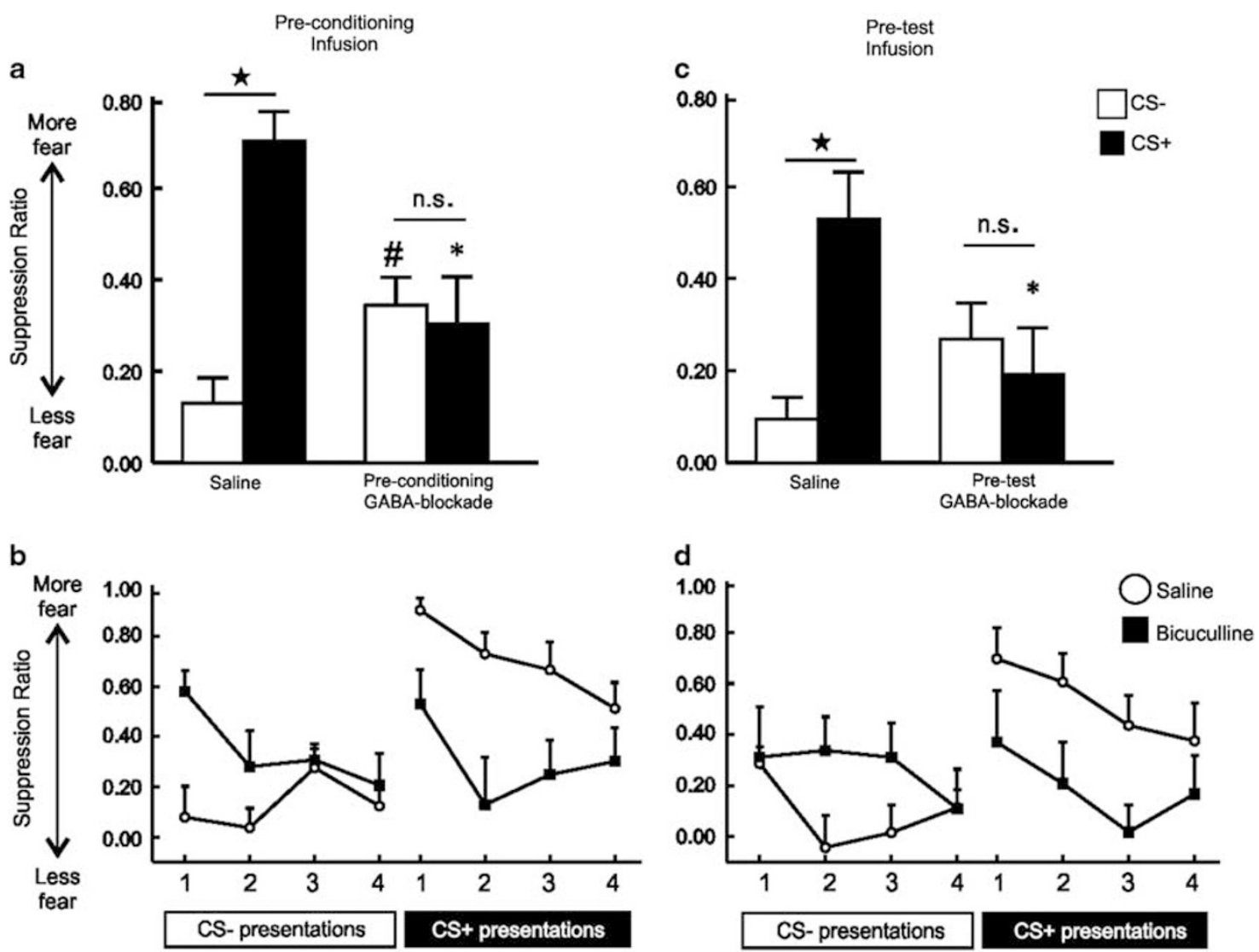

Figure 2 PFC GABA transmission regulates acquisition and expression of discriminative fear conditioning. Graphs depict average suppression (across four CS presentations) for animals receiving $(a, b)$ pre-conditioning or $(c, d)$ pre-test infusion on a discriminative fear conditioning task. Error bars represents +SEM. (a) Saline-infused animals displayed elevated fear to the CS + and decreased fear to the CS-. However, animals receiving bicuculline infusions before conditioning did not display such discrimination, instead showing elevated fear to a CS-and decreased fear to a CS + relative to controls. (b) Salineinfused animals showed discrimination across all four CS-/CS + tones (open circles), while pre-conditioning GABA-blockade animals did not (black squares). (c) Intra mPFC infusions of bicuculline before the discriminative fear test also abolished discrimination between the CS + and CS-. (d) Saline-infused animals showed discrimination across all four CS-/CS + tones (open circles), while pre-test GABA-blockade animals did not (black squares). Black star denotes $p<0.05$ as compared with CS-. ${ }^{\#}$ Denotes $p<0.05$ between CS- for saline and pre-conditioning GABA-blockade. *Denotes $p<0.05$ vs saline. 
measure for bicuculline-treated rats (NS). Furthermore, PFC GABA-blockade during conditioning led to elevated fear during presentation of the CS- $(p<0.05)$, while at the same time decreasing fear in response to the CS + , compared with saline controls $(p<0.001$, Figure $2 \mathrm{a})$. There was no significant main effect of Treatment, $(F(2,43)=2.10$, NS), indicating that over the entire test session, rats in both groups displayed a comparable amount of conditioned suppression, although the manner in which rats distributed their fear response to the CS $+v s$ CS- was radically different between treatment groups. Similarly, there was no significant three-way interaction, $(F(3,69)=1.48$, NS; Figure $3 b)$. In addition, there was no effect of drug on locomotion, $(t(23)=-0.454$, NS), or the rates of lever pressing, $(t(23)=-0.137, \mathrm{NS} ;$ Table 1$)$. One animal included in the above analysis had a placement that encroached ventrally into the prelimbic/infralimbic mPFC border. Conditioned suppression data from this animal were comparable to those with placements exclusively within the prelimbic cortex $(\mathrm{CS}-=0.44, \mathrm{CS}+=0.33)$. Collectively, these data show that reducing PFC GABA activity during acquisition of discriminative conditioned fear causes a disruption in the expression of stimulus-appropriate emotional responses, increasing fear in response to a neutral stimulus and reducing fear to an aversive one.

Pre-test manipulations. Similar to what was observed in the pre-conditioning experiment, saline-treated control rats $(n=10)$ showed robust discrimination between the aversive $\mathrm{CS}+$ and a non-aversive CS- (Figure 2c, right). Conversely, rats subjected to the conditioning phase drug-free followed by intra-mPFC infusions of bicuculline before test $(n=11)$ again displayed impaired discrimination, as revealed by a significant Treatment $\times$ Stimulus type interaction, $(F(1,19)=$ 10.97, $p=<0.01$; Figure 2c). Simple-main effects analyses confirmed that control rats showed substantially greater suppression during CS $+v s$ CS- presentations $(p<0.01$; Figure $2 \mathrm{c}$ ), but there was no difference on this measure in bicuculline-treated rats (NS). In this experiment, the enhanced suppression in response to CS- presentation in bicuculline-treated rats was only trend level when compared with controls $(p=0.087)$, although the drug group did show a significant decrease in fear to the CS $+(p<0.05$, Figure 2c). There was no significant main effect of treatment or three-way interaction, (all $F s<1.1$, NS; Figure 2d). Similarly, there were no differences between groups in terms of locomotion or on the rate of lever pressing, (all t's(19)<1.4, NS; Table 1). As in the pre-conditioning experiment, one cannula placement from one animal encroached into the prelimbic/infralimbic mPFC border. This animal displayed elevated fear to the CS- (suppression ratio $=0.80$ ), with no corresponding decrease in fear to the $\mathrm{CS}+($ suppression ratio $=0.59)$. Further characterization of subregional contribution would be necessary to tease apart potential differential prelimbic and infralimbic contribution. Thus, disruption of mPFC GABA signaling during recall disrupts discriminative control over conditioned fear responses, although in this instance, the effect is driven more prominently by reduced fear expression to an aversive stimulus.

\section{Latent Inhibition}

As displayed in Figure 3a, control animals showed the classic LI effect, whereby PE animals $(n=8)$ suppressed lever pressing less than NPE $(n=8)$ animals. This effect was apparent only during the first CS presentation, as the suppression in the NPE group extinguished during subsequent CS presentations. Therefore, in order to analyze

Table I Mean ( \pm SEM) Values for Total Locomotion and Rate of Lever Pressing Following mPFC GABA-Blockade on a

Discriminative Fear Conditioning Task

\begin{tabular}{llcc}
\hline Infusion timeline & Treatment & $\begin{array}{c}\text { Locomotion } \\
\text { (photobeam breaks) }\end{array}$ & $\begin{array}{c}\text { Lever } \\
\text { presses/min }\end{array}$ \\
\hline Pre-conditioning & Saline & $|37|( \pm 167)$ & $16.9( \pm 1.6)$ \\
& Bicuculline & $\mid 274( \pm 139)$ & $16.5( \pm 2.0)$ \\
Pre-test & Saline & $1044( \pm 116)$ & $16.5( \pm 3.2)$ \\
& Bicuculline & $1356( \pm 238)$ & $11.1( \pm 2.2)$ \\
\hline
\end{tabular}
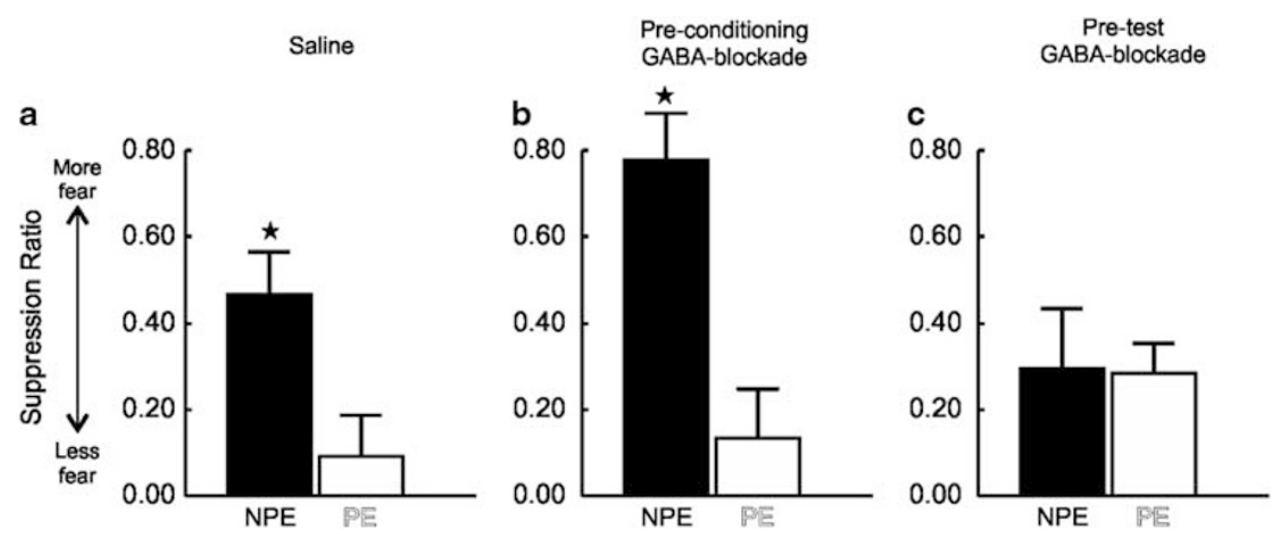

Figure 3 Reducing PFC GABA transmission disrupts $L I$ of conditioned fear expression. Suppression data for animals receiving intra-mPFC saline before both conditioning and test phases (a) or bicuculline before conditioning (b) or test (c). All error bars reflect + SEM. (a) Saline-infused animals displayed a classic LI effect, with PE animals showing less fear than NPE animals during test. (b) Pre-conditioning bicuculline infusion did not alter LI expression, as the NPE group displayed elevated fear as compared with the PE group. (c) LI was abolished by pre-test intra-mPFC bicuculline, with there being no difference in fear between NPE and PE animals in these conditions. Star denotes $p<0.05$ between PE and NPE groups. 
alterations in LI specifically across the groups, our initial analysis focused on conditioned suppression during the first CS presentation, as has been done in prior studies using this LI procedure (McAllister, 1997). Analysis of these data revealed a significant main effect of $\mathrm{PE} / \mathrm{NPE}$, $(\mathrm{F}(1,43)=15.60, p<0.001)$, no main effect of Treatment, $(\mathrm{F}(2,43)=1.76$, NS), but importantly, also uncovered a significant Treatment by $\mathrm{PE} / \mathrm{NPE}$ interaction, $(\mathrm{F}(2,43)=$ $4.46, p<0.05)$. Simple-main effects analyses confirmed that NPE control rats displayed greater levels of conditioned suppression relative to PE control rats $(p<0.05$, Figure $3 a)$. Similarly, rats that received infusions of the GABA-receptor antagonist before conditioning demonstrated LI, with NPE rats $(n=9)$ showing more fear compared with their PE $(n=8)$ counterparts $(p<0.001$, Figure $3 \mathrm{~b})$. In addition, NPE rats treated with bicuculline during conditioning displayed higher levels of suppression relative to NPE control rats, although comparison of these two values only yielded a trend toward statistical significance $(p<0.076$, Figures 3a and $b$, black bars). In stark contrast to the other treatment groups, intra-mPFC GABA-blockade before the test session completely abolished the LI effect, wherein both NPE $(n=8)$ and PE $(n=8)$ animals displayed comparable levels of conditioned fear to the first CS presentation (NS, Figure 3c). Thus, disruption of mPFC GABA signaling during PE/conditioning does not affect the acquisition of LI, but this manipulation before test markedly disrupted the recall of learned irrelevance.

We then conducted a second analysis comparing conditioned suppression over all four-tone presentations, to ascertain whether there were any differences between groups in the subsequent expression and extinction of conditioned fear (Figure 4). This analysis revealed a significant Treatment $\times \mathrm{PE} / \mathrm{NPE}$ interaction, $(\mathrm{F}(2,43)=3.43, p<0.05)$, although the three-way interaction was not significant, $(\mathrm{F}(6,129)=0.82$, NS). Subsequent partitioning of the twoway interaction revealed that, for PE animals, there were no differences in the levels of conditioned suppression across the treatment groups, $(\mathrm{F}(2,21)=0.82$, NS; Figure 4a). However, for NPE animals, bicuculline treatment before the conditioning phase significantly exacerbated the fear response, when compared with controls or rats receiving bicuculline before the LI test, which did not differ from each other $(\mathrm{F}(2,43)=3.43, p<0.05$ and Tukey's, $p<0.05)$. There was no difference in locomotion across NPE or PE conditions, or treatment groups, and no interaction (all $F$ 's $<3.9$, all $p$ 's $>0.05$; Table 2). There was a slight decrease in the rates of lever pressing for rats receiving bicuculline prior the test session for LI, $(\mathrm{F}(2,43)=3.81, p<0.05$, Tukey's, $p<0.05$ ), as compared with controls (Table 2). Collectively, these data indicate that disruption of mPFC GABA transmission during distinct phases of learning has differential effects on acquisition and LI of conditioned fear. Reducing mPFC GABA impairs the recall of learned irrelevance, whereas disinhibition of the $\mathrm{MPFC}$ during standard non-discriminative fear conditioning leads to exaggerated expression of a fear response.

\section{DISCUSSION}

The present findings reveal a critical role for mPFC GABA in regulating different aspects of aversive conditioning, suggesting that normal inhibitory transmission in this region is required for the appropriate utilization of affective information. Using a translational discriminative fear assay, we observed that pharmacological reduction of mPFC $\mathrm{GABA}_{\mathrm{A}}$ signaling eliminated the ability to discriminate between a neutral CS- and an aversive CS + . Disruption of mPFC GABA activity also induced dissociable effects on the acquisition and expression of conditioned fear within the context of a LI task. Intra-mPFC infusions of bicuculline

Table 2 Mean ( \pm SEM) Values for Total Locomotion and Rate of Lever Pressing Following mPFC GABA-Blockade During Tests of Latent Inhibition

\begin{tabular}{lcc}
\hline Treatment & $\begin{array}{c}\text { Locomotion } \\
\text { (photobeam breaks) }\end{array}$ & Lever presses/min \\
\hline Controls & $927( \pm 124)$ & $29.11( \pm 2.9)$ \\
Pre-conditioning Bicuculline & $1007( \pm 83)$ & $21.80( \pm 2.8)$ \\
Pre-test bicuculline & $977( \pm 105)$ & $17.98( \pm 2.9)^{\mathrm{a}}$ \\
\hline
\end{tabular}

$a_{p}<0.05$ vs saline.
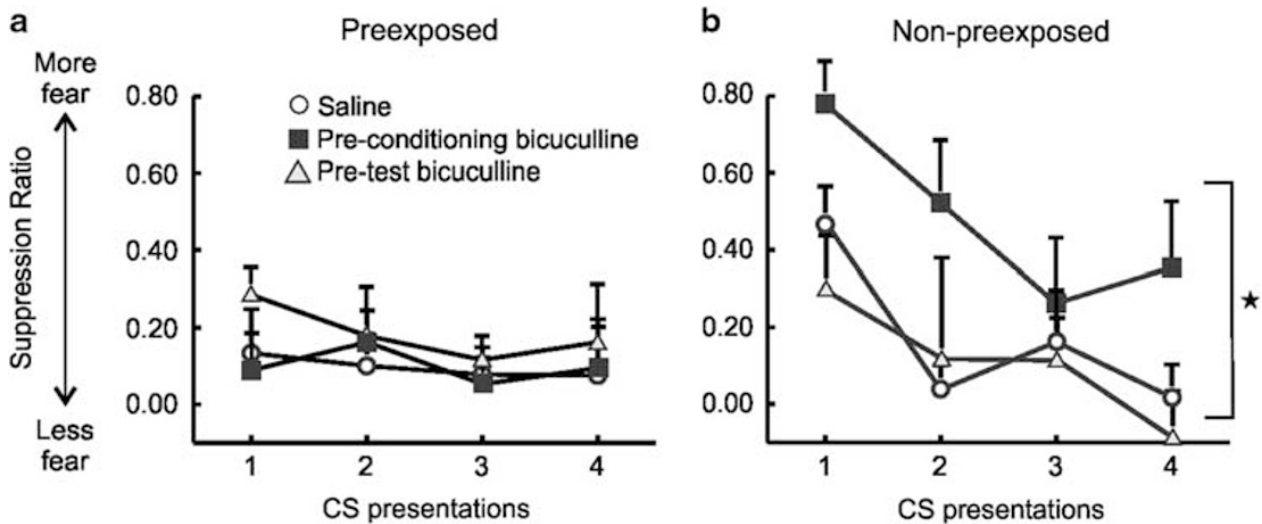

Figure 4 Reducing PFC GABA transmission during fear conditioning enhances expression of learned fear. All error bars reflect + SEM. (a) In rats that were preexposed to the CS without shocks, there were no differences in conditioned suppression between the three groups. (b) In contrast, in nonpreexposed rats, intra-mPFC bicuculline before conditioning (black squares) resulted in elevated fear expression across four tones as compared with control animals (open circles) or pre-test bicuculline (gray triangle) infused animals. Star denotes $p<0.05$ pre-conditioning bicuculline vs the other groups. 
during conditioning spared LI, but markedly augmented fear expression in a NPE group. In contrast, reducing GABA transmission during the LI test abolished the LI effect, pointing to a key role for this system in mediating the recall of learned irrelevance. These results serve to illuminate the importance of normal prefrontal GABA signaling in modulating aversive learning, and suggest that dysfunction of such signaling may underlie emotional perturbations and inappropriate use of affective information.

\section{PFC GABA Regulation of Discriminative Fear Conditioning}

Much of what is known about the role of the PFC in fear conditioning comes from classic, single-stimulus (nondiscriminative) paradigms, which generally illustrate that the prelimbic mPFC is critical for fear expression (BurgosRobles et al, 2009; Vidal-Gonzalez et al, 2006). Here we provide evidence that a disinhibited or 'noisy' mPFC impairs aversive Pavlovian discrimination learning when induced before the acquisition or expression phase of the task. Previous research suggests that acquisition and expression of aversive discriminative Pavlovian or avoidance learning is dependent on the integrity of the mPFC (Frysztak and Neafsey, 1991, 1994; Gabriel and Orona, 1982; Gibbs and Powell, 1991; Maxwell et al, 1994; Orona and Gabriel, 1983). Recent studies have shown that during discriminative fear learning, activity within the basolateral amygdala (BLA; a region critical for fear learning) becomes entrained to theta frequency input from the MPFC (Likhtik et al, 2014). Interestingly, these processes appears to be critically dependent on the inhibition of a sub-population of mPFC pavalbumin-positive GABAergic interneurons (Courtin et al, 2013). Fear discrimination is then associated with the temporally coordinated disinhibition of PFC projection neurons, which influence BLA activity and subsequent fear expression. Thus, well-orchestrated activity in the $\mathrm{mPFC}$ is likely necessary for appropriate discrimination between stimuli that are explicitly aversive or neutral. As PFC GABAergic antagonism is known to increase activity of PFC neurons non-selectively (Lodge, 2011; Paine et al, 2011; Rao et al, 2000), this would be expected to result in an impairment in discriminative neural activity and consequent behavior. Consistent with this interpretation, animals responded essentially identically regardless of the aversive/ neutral nature of the particular CS presented during the test phase. The resulting fear-related suppression was not simply generalized (high or low fear to both CS +/CS-), but instead markedly lower fear to the CS + and elevated fear to the CS-. Thus, the tuning of mPFC circuitry by $\mathrm{GABA}_{\mathrm{A}}$-receptor-mediated transmission may be critical for the appropriate encoding and recall of information as either emotionally salient or innocuous.

Alterations in affective salience attribution have also been observed following dopaminergic manipulation of the mPFC in a manner consistent with its ability to modulate inhibitory transmission (Lauzon and Laviolette, 2010; Lauzon et al, 2009; Laviolette et al, 2005). For example, mPFC $\mathrm{D}_{4}{ }^{-}$ receptor stimulation enhances the affective salience of a stimulus that control animals do not find affectively salient, while also dampening the salience of a stimulus that normally possesses such salience (Lauzon et al, 2009, 2012).
Interestingly, $\mathrm{D}_{4}$-receptor blockade eliminates the ability of $\mathrm{mPFC}$ neurons to encode the affective importance of an aversively CS (Laviolette et al, 2005). Dopamine is known to tune prefrontal excitatory/inhibitory tone, with $\mathrm{D}_{4}$ receptors thought to regulate GABA interneuron activity (Floresco and Tse, 2007; Onn et al, 2006; Yuen and Yan, 2009). Although $D_{4}$ receptors are also localized on glutamatergic pyramidal neurons, Lauzon and Laviolette (2010) have proposed that $\mathrm{D}_{4}$-receptor stimulation may disinhibit pyramidal cell output via inhibition of interneurons. Any changes in $\mathrm{mPFC}$ dopamine tone would be expected to alter inhibitory transmission, and subsequent affective salience attributions. The present findings support the idea that prefrontal dopamine influences salience attribution through the modulation of GABAergic transmission.

\section{PFC GABA Regulation of LI}

During LI, PE subjects learn about the irrelevance of a preexposed stimulus, as reflected by slower learning of subsequent associations between that CS and unconditioned stimuli. The majority of studies investigating the role of the mPFC in LI have generally observed that permanent lesions of this structure do not affect LI (Joel et al, 1997; Lacroix et al, 2000; Schiller and Weiner, 2004). In these studies, lesions were induced before the conditioning and PE phases, making it difficult to assess the relative contribution of the region to discrete task phases, particularly recall/expression during the test phase. In contrast to these previous reports, we observed a previously uncharacterized role for $\mathrm{MPFC}$ GABA transmission in mediating the expression of LI, as blockade of $\mathrm{GABA}_{\mathrm{A}}$ receptors before an expression test abolished the LI effect. Thus, whereas elimination of PFC outflow via lesions may not have a deleterious effect on the acquisition/expression of LI, the present results show that the recall of learned irrelevance is dependent on intact GABAergic transmission within the mPFC.

To our knowledge, the present findings are a rare demonstration that manipulations administered during tests of LI can disrupt the retrieval of learned irrelevance (Lewis and Gould, 2007). This being the case, the particular mechanisms underlying the abolition of LI following pretest mPFC GABA-blockade is unknown. Although the electrophysiological correlates of LI are poorly characterized, one study has shown that PE to a CS in a LI procedure led to attenuation of CS-evoked neural responses of $\mathrm{mPFC}$ neurons (Talk et al, 2005). Such 'neural LI' was observed in every other region investigated, including the amygdala, thalamus, and substantia nigra, suggesting that this physiological signature may be ubiquitous across brain regions. Thus, disinhibition of the mPFC may disrupt the appropriate recall of CS associations, and consequently LI, by eliminating the adaptive encoding of neural LI in the MPFC or other downstream regions that regulate this aspect of learning, such as the nucleus accumbens shell (Gal et al, 2005) or entorhinal cortex (Lewis and Gould, 2007). Additional research into the neural mechanisms underlying the expression and recall of learned irrelevance may shed additional light on this issue.

Unlike the above-mentioned findings, intra-mPFC infusions of bicuculline before conditioning did not alter LI, suggesting that mPFC GABA signaling is not critical for 
learning about the irrelevance of a PE stimulus, as we have shown previously (Enomoto et al, 2011). However, in NPE rats that received a relatively mild fear conditioning protocol (no PE; three CS/shock pairings), mPFC GABA antagonism during conditioning markedly enhanced subsequent expression of conditioned fear across the four CS exposures, a finding that meshes with the established role for the prelimbic mPFC in these processes (Sotres-Bayon and Quirk, 2010). Presentation of an aversive CS increases neural activity in the prelimbic $\mathrm{mPFC}$ in a manner that is correlated with extinction failures, with greater activity associated with slower fear extinction (Burgos-Robles et al, 2009). Similarly, prelimbic mPFC microstimulation elevates conditioned fear (Vidal-Gonzalez et al, 2006), whereas inactivation impairs its expression (Sierra-Mercado et al, 2011). Note that inactivation of the MPFC during acquisition does not affect expression of conditioned fear (Corcoran and Quirk, 2007). Nevertheless, the present data highlight another previously uncharacterized role for $\mathrm{MPFC}$ GABA in aversive Pavlovian conditioning. In the absence of stimulus PE, disinhibition of the MPFC during acquisition of CS/shock associations appears to augment encoding of a fear memory, leading to elevated fear expression. At first glance, the enhanced fear induced by mPFC GABA-blockade in NPE rats may seem at odds with our observation that similar treatments during discriminative fear conditioning reduced fear to a $\mathrm{CS}+$. However, it is important to emphasize that mPFC GABA antagonism also enhanced fear evoked by a CS- not paired with shock. Indeed, when collapsed over the entire test session, bicuculline-treated rats showed the same average level of conditioned suppression as controls, but the manner in which fear was allocated to the CS + and CS- was markedly different. This suggests that treatment caused the formation of nonspecific associations with the shock that generalized to both CSs. When juxtaposed, the findings from these two experiments imply that $\mathrm{mPFC}$ GABA transmission aids in refining how Pavlovian fear memories are encoded, either mitigating the relative strength of a fear memory to a single CS or allocating appropriate fear or safety associations to different stimuli that may or may not be associated with aversive events. In this regard, there is evidence to suggest that patterns of mPFC activity that encode aversive and safety signals differ considerably from those that encode a single aversive CS. During discriminative fear learning, activity of separate populations of $\mathrm{mPFC}$ neurons reflect the safety of the CS- and aversive nature of a CS + (Maxwell et al, 1994). In comparison, during conditioning of a single stimulus, most prelimbic mPFC neurons are excited by an aversive CS (Baeg et al, 2001; Gilmartin and McEchron, 2005). As such, it is reasonable to propose that disinhibition of $\mathrm{mPFC}$ activity during discriminative fear learning (as may be induced by pharmacological reduction of GABA transmission) would interfere with the distinct patterns of activity in separate neuronal populations that encode the CS- or CS + , leading to generalization of a fear response across stimuli. On the other hand, disinhibition of a majority of PFC neurons that encode a single aversive CS could augment encoding of the aversive nature of the CS, manifesting in elevated fear that delays extinction.

Disinhibition of the mPFC may differentially alter the acquisition of salience during aversive conditioning through effects on downstream structures implicated in the acquisition, expression, and LI of conditioned fear. In particular, activation of the BLA may be one site that drives these effects. The BLA is critical for the formation of CSunconditioned stimulus associations and receives monosynaptic projections from the prelimbic mPFC. mPFC-BLA synchrony increases during discriminative fear conditioning (Likhtik et al, 2014), and imaging studies have shown that activation of the BLA differentiates between aversive and non-aversive stimuli during conditioning (McHugh et al, 2013). Disinhibition of the PFC and the resultant excitatory outflow to the BLA would be expected to perturb this type of discriminative activity. In comparison, during standard, single-stimulus fear-conditioning, computational models predict that prelimbic $\mathrm{mPFC}$ may drive BLA activity to signal fear (Pendyam et al, 2013), a process that could be enhanced following $\mathrm{mPFC}$ disinhibition. Moreover, lesions of the BLA lead to abnormally persistent LI (Schiller and Weiner, 2004). As such, it is plausible that enhanced activity of the BLA may produce the opposite effect, attenuating LI expression.

The present findings converge with an emerging literature demonstrating that mPFC GABA-blockade can impair various cognitive functions, including attention and cognitive flexibility (Enomoto et al, 2011; Paine et al, 2011; Pehrson et al, 2013). With respect to attention, it is unlikely that impairments in these aspects of cognition can explain the totality of the results reported here. Inattention during discriminative fear conditioning would be expected to manifest itself as decreased fear to both stimuli during test. Instead, rats paradoxically showed elevated fear to the CS-, and lower fear to the CS +. Further arguments against an attentional mechanism come from the fact that LI is an index of learned irrelevance or inattention. Yet, LI is unaffected by mPFC GABAergic-blockade during PE/conditioning (ie, the phase of the procedure that places greatest demands on attention, Enomoto et al, 2011; this study). On the other hand, reducing PFC GABA transmission during the acquisition of a visual discrimination led to increased perseveration when rats were required to shift to using a different discrimination strategy, suggesting that these treatments may have augmented the formation or consolidation of certain action-outcome associations (Enomoto et al, 2011). A similar mechanism may underlie the enhanced Pavlovian fear associations induced by bicuculline infusions during conditioning in NPE rats. However, impairments in cognitive flexibility would not readily explain impairments in discriminative fear, as in this instance, rats showed comparable levels of fear to both a $\mathrm{CS}+$ and CS-, which could be interpreted as a more flexible allocation of affective responding. Thus, although disruption in other aspects of cognition induced by PFC GABAblockade may have contributed to some of the effects observed in this study, we suggest that these deficits are more reflective of perturbations in the attributions of affective salience during aversive conditioning.

\section{Relevance for Schizophrenia}

Our finding that mPFC GABA activity has a critical role in modulating various aspects of aversive learning provides novel insight into the mechanisms underlying emotional 
disturbances that may occur when this system is dysfunctional, as may be the case in schizophrenia. For example, Jensen et al (2008) assessed fear conditioning in schizophrenic patients and non-psychiatric controls, requiring them to discriminate between an aversive CS + and a neutral CS-, similar to the assay used in this study. Compared with healthy controls, patients displayed less physiological and subjective arousal to a CS + and more arousal to a CS-, an effect that is nearly identical to the deficits in discriminative fear reported here. Similar results have been obtained from individuals high in the schizotypal trait 'reality distortion' (Balog et al, 2013), suggesting that these deficits may be related to underlying neurochemical (possibly GABAergic) alterations, rather than confounding factors such as disease course or antipsychotic treatment. Interestingly, the mPFC displays aberrant hyperactivity in response to neutral stimulus in schizophrenia patients compared with control individuals (Hall et al, 2008; Jensen et al, 2008; Murray et al, 2008). The present findings suggest that these neural and corresponding emotional abnormalities may be driven in part by perturbations in PFC GABAergic activity.

Deficient LI in schizophrenia has been proposed to be mediated by elevated striatal dopamine transmission that may lead to aberrant attributions of salience to an otherwise irrelevant stimulus (Weiner and Arad, 2009; Weiner, 2003). In this study, PFC GABA-blockade during conditioning/PE left LI intact, despite the fact that MPFC GABA-blockade enhances phasic activity of midbrain dopamine neuron activity (Enomoto et al, 2011). This lack of effect suggests that increased phasic firing of dopamine neurons induced by PFC GABA-blockade may not be sufficient to elevate striatal dopamine levels to the extent required to disrupt LI. Alternatively, it may be that increases in tonic, rather than phasic dopamine transmission may be required to perturb LI, as we have argued previously (Enomoto et al, 2011). In contrast, pre-test reductions in PFC GABA transmission abolished expression of LI, indicating that intact PFC GABA activity is essential for retrieving information about the irrelevance of stimuli. It is therefore reasonable to propose that, in addition to aberrant increases in dopamine activity, perturbations in PFC GABA transmission that impede the recall of learned irrelevance may also contribute to impairments in LI observed in schizophrenia. On the other hand, mPFC GABA-blockade during acquisition of conditioned fear to a single stimulus in NPE animals increased the expression of conditioned fear during test. This exacerbated fear response is reminiscent of deficient extinction learning in schizophrenia, whereby individuals do not recall extinction memories as strongly as controls and continuing to display elevated conditioned responses despite extinction training (Holt et al, 2009, 2012). In this regard, extinction failure is correlated with ventromedial PFC overactivation in schizophrenia patients. These changes are particularly interesting given that schizophrenia is highly comorbid with anxiety disorders (Braga et al, 2004; Buckley et al, 2009), which have also been associated with decreased prefrontal GABA activity (Long et al, 2013; Simpson et al, 2012). The present findings suggest that these abnormalities may be related to changes in excitation/inhibition because of endemic PFC GABAergic dysfunction.

Although in vivo evidence for PFC GABA hypofunction in schizophrenia remains controversial (Ongür et al, 2010;
Rowland et al, 2013), a recent report by Taylor et al (2013) suggests that endemic cortical GABA dysfunction may contribute to abnormal neural responses to affective stimuli. In that study, the authors reported that schizophrenic individuals treated with lorazepam (a positive allosteric modulator of the GABA-receptor benzodiazepine binding site), displayed increased activity in the dorsomedial PFC in response affectively salient stimuli, whereas these same treatments decreased PFC activity in controls. These differential effects of pharmacological GABAergic challenge on PFC activity associated with emotional processing further support the contention that dysfunction in this system may underlie disturbances in affective regulation observed in schizophrenia, and indicate that further research on how normal and abnormal PFC GABA transmission regulates these processes is warranted.

\section{Summary and Conclusions}

This study reveals that PFC GABA neurotransmission has multiple and previously uncharacterized roles in regulating different aspects of conditioned fear. Pharmacological reductions in PFC GABA transmission impaired discriminative aversive conditioning, retrieval of LI and exacerbated fear learning to a single stimulus. Each of these alterations in emotional learning resemble those that have been observed in schizophrenic patients. As such, these findings suggest that dysfunction within PFC GABAergic circuits may underlie abnormal affect regulation associated with this disorder such as schizophrenia, and that development of treatments that may normalize PFC GABA activity may be beneficial in ameliorating emotional abnormalities associated with the disease.

\section{FUNDING AND DISCLOSURE}

SBF has received funds to conduct contract work for Pfizer. The work supported by this grant is not related to the findings presented in this paper. The remaining author declares no conflict of interest.

\section{ACKNOWLEDGEMENTS}

This research was supported by grants from the Canadian Institutes of Health Research to SBF (MOP-130393). PTP is the recipient of a University of British Columbia Graduate Fellowship.

\section{REFERENCES}

Akbarian S, Kim JJ, Potkin SG, Hagman JO, Tafazzoli A, Bunney WE et al (1995). Gene expression for glutamic acid decarboxylase is reduced without loss of neurons in prefrontal cortex of schizophrenics. Arch Gen Psychiatry 52: 258-266.

Anticevic A, Repovs G, Corlett PR, Barch DM (2011). Negative and nonemotional interference with visual working memory in schizophrenia. Biol Psychiatry 70: 1159-1168.

Antunes R, Moita MA (2010). Discriminative auditory fear learning requires both tuned and nontuned auditory pathways to the amygdala. J Neurosci 30: 9782-9787.

Asinof SK, Paine TA (2013). Inhibition of GABA synthesis in the prefrontal cortex increases locomotor activity but does not affect 
attention in the 5-choice serial reaction time task. Neuropharmacology 65: 39-47.

Baeg EH, Kim YB, Jang J, Kim HT, Mook-Jung I, Jung MW (2001). Fast spiking and regular spiking neural correlates of fear conditioning in the medial prefrontal cortex of the rat. Cereb Cortex 11: 441-451.

Balog Z, Somlai Z, Kéri S (2013). Aversive conditioning, schizotypy, and affective temperament in the framework of the salience hypothesis. Pers Individ Dif 54: 109-112.

Bang SJ, Allen TA, Jones LK, Boguszewski P, Brown TH (2008). Asymmetrical stimulus generalization following differential fear conditioning. Neurobiol Learn Mem 90: 200-216.

Benes FM, Berretta S (2001). GABAergic interneurons: implications for understanding schizophrenia and bipolar disorder. Neuropsychopharmacology 25: 1-27.

Braga RJ, Petrides G, Figueira I (2004). Anxiety disorders in schizophrenia. Compr Psychiatry 45: 460-468.

Buckley PF, Miller BJ, Lehrer DS, Castle DJ (2009). Psychiatric comorbidities and schizophrenia. Schizophr Bull 35: 383-402.

Burgos-Robles A, Vidal-Gonzalez I, Quirk GJ (2009). Sustained conditioned responses in prelimbic prefrontal neurons are correlated with fear expression and extinction failure. J Neurosci 29: 8474-8482.

Corcoran KA, Quirk GJ (2007). Activity in prelimbic cortex is necessary for the expression of learned, but not innate, fears. J Neurosci 27: 840-844.

Courtin J, Chaudun F, Rozeske RR, Karalis N, Gonzalez-Campo C, Wurtz $\mathrm{H}$ et al (2013). Prefrontal parvalbumin interneurons shape neuronal activity to drive fear expression. Nature 505: 92-96.

Diaconescu AO, Jensen J, Wang H, Willeit M, Menon M, Kapur S et al (2011). Aberrant effective connectivity in schizophrenia patients during appetitive conditioning. Front Hum Neurosci 4: $1-14$.

Enomoto T, Tse MT, Floresco SB (2011). Reducing prefrontal gamma-aminobutyric acid activity induces cognitive, behavioral, and dopaminergic abnormalities that resemble schizophrenia. Biol Psychiatry 69: 432-441.

Floresco SB, Tse MT (2007). Dopaminergic regulation of inhibitory and excitatory transmission in the basolateral amygdalaprefrontal cortical pathway. J Neurosci 27: 2045-2057.

Frysztak RJ, Neafsey EJ (1991). The effect of medial frontal cortex lesions on respiration, 'freezing,' and ultrasonic vocalizations during conditioned emotional responses in rats. Cereb Cortex 1: 418-425.

Frysztak RJ, Neafsey EJ (1994). The effect of medial frontal cortex lesions on cardiovascular conditioned emotional responses in the rat. Brain Res 643: 181-193.

Gabriel M, Orona E (1982). Parallel and serial processes of the prefrontal and cingulate cortical systems during behavioral learning. Brain Res Bull 8: 781-785.

Gal G, Schiller D, Weiner I (2005). Latent inhibition is disrupted by nucleus accumbens shell lesion but is abnormally persistent following entire nucleus accumbens lesion: the neural site controlling the expression and disruption of the stimulus preexposure effect. Behav Brain Res 162: 246-255.

Gibbs CM, Powell DA (1991). Single-unit activity in the dorsomedial prefrontal cortex during the expression of discriminative bradycardia in rabbits. Behav Brain Res 43: 79-92.

Gilmartin MR, McEchron MD (2005). Single neurons in the medial prefrontal cortex of the rat exhibit tonic and phasic coding during trace fear conditioning. Behav Neurosci 119: $1496-1510$.

Gonzalez-Burgos G, Hashimoto T, Lewis DA (2010). Alterations of cortical GABA neurons and network oscillations in schizophrenia. Curr Psychiatry Rep 12: 335-344.

Gonzalez-Burgos G, Lewis DA (2012). NMDA receptor hypofunction, parvalbumin-positive neurons and cortical gamma oscillations in schizophrenia. Schizophr Bull 38: 950-957.
Gray NS, Pilowsky LS, Gray JA, Kerwin RW (1995). Latent inhibition in drug naive schizophrenics: relationship to duration of illness and dopamine D2 binding using SPET. Schizophr Res 17: 95-107.

Gray NS, Snowden RJ (2005). The relevance of irrelevance to schizophrenia. Neurosci Biobehav Rev 29: 989-999.

Hall J, Whalley HC, McKirdy JW, Romaniuk L, McGonigle D, McIntosh AM et al (2008). Overactivation of fear systems to neutral faces in schizophrenia. Biol Psychiatry 64: 70-73.

Holt DJ, Coombs G, Zeidan MA, Goff DC, Milad MR (2012). Failure of neural responses to safety cues in schizophrenia. Arch Gen Psychiatry 69: 893-903.

Holt DJ, Lebron-Milad K, Milad MR, Rauch SL, Pitman RK, Orr SP et al (2009). Extinction memory is impaired in schizophrenia. Biol Psychiatry 65: 455-463.

Jensen J, Willeit M, Zipursky RB, Savina I, Smith AJ, Menon M et al (2008). The formation of abnormal associations in schizophrenia: neural and behavioral evidence. Neuropsychopharmacology 33: 473-479.

Joel D, Weiner I, Feldon J (1997). Electrolytic lesions of the medial prefrontal cortex in rats disrupt performance on an analog of the Wisconsin Card Sorting Test, but do not disrupt latent inhibition: implications for animal models of schizophrenia. Behav Brain Res 85: 187-201.

Kamin LJ, Brimer CJ, Black AH (1963). Conditioned suppression as a monitor of fear of the CS in the course of avoidance training. J Comp Phyisological Psychol 56: 497-501.

Kapur S (2003). Psychosis as a state of aberrant salience: a framework linking biology, phenomenology, and pharmacology in schizophrenia. Am J Psychiatry 160: 13-23.

Lacroix L, Spinelli S, White W, Feldon J (2000). The effects of ibotenic acid lesions of the medial and lateral prefrontal cortex on latent inhibition, prepulse inhibition and amphetamineinduced hyperlocomotion. Neuroscience 97: 459-468.

Lauzon NM, Ahmad T, Laviolette SR (2012). Dopamine D4 receptor transmission in the prefrontal cortex controls the salience of emotional memory via modulation of calcium calmodulin-dependent kinase II. Cereb Cortex 22: 2486-2494.

Lauzon NM, Bishop SF, Laviolette SR (2009). Dopamine D1 versus D4 receptors differentially modulate the encoding of salient versus nonsalient emotional information in the medial prefrontal cortex. J Neurosci 29: 4836-4845.

Lauzon NM, Laviolette SR (2010). Dopamine D4-receptor modulation of cortical neuronal network activity and emotional processing: implications for neuropsychiatric disorders. Behav Brain Res 208: 12-22.

Laviolette SR, Lipski WJ, Grace AA (2005). A subpopulation of neurons in the medial prefrontal cortex encodes emotional learning with burst and frequency codes through a dopamine D4 receptor-dependent basolateral amygdala input. J Neurosci 25: 6066-6075.

Lewis DA, Curley AA, Glausier JR, Volk DW (2012). Cortical parvalbumin interneurons and cognitive dysfunction in schizophrenia. Trends Neurosci 35: 57-67.

Lewis DA, Volk DW, Hashimoto T (2004). Selective alterations in prefrontal cortical GABA neurotransmission in schizophrenia: a novel target for the treatment of working memory dysfunction. Psychopharmacology (Berl) 174: 143-150.

Lewis MC, Gould TJ (2007). Signal transduction mechanisms within the entorhinal cortex that support latent inhibition of cued fear conditioning. Neurobiol Learn Mem 88: 359-368.

Likhtik E, Stujenske JM, Topiwala MA, Harris AZ, Gordon JA (2014). Prefrontal entrainment of amygdala activity signals safety in learned fear and innate anxiety. Nat Neurosci 17: $106-113$.

Lodge DJ (2011). The medial prefrontal and orbitofrontal cortices differentially regulate dopamine system function. Neuropsychopharmacology 36: 1227-1236. 
Long Z, Medlock C, Dzemidzic M, Shin Y-W, Goddard AW, Dydak U (2013). Decreased GABA levels in anterior cingulate cortex/ medial prefrontal cortex in panic disorder. Prog Neuropsychopharmacol Biol Psychiatry 44: 131-135.

Maxwell B, Powell DA, Buchanan SL (1994). Multiple- and singleunit activity in area 32 (prelimbic region) of the medial prefrontal cortex during Pavlovian heart rate conditioning in rabbits. Cereb Cortex 4: 230-246.

McAllister KH (1997). A single administration of d-amphetamine prior to stimulus pre-exposure and conditioning attenuates latent inhibition. Psychopharmacology (Berl) 130: 79-84.

McHugh SB, Marques-Smith A, Li J, Rawlins JNP, Lowry J, Conway $M$ et al (2013). Hemodynamic responses in amygdala and hippocampus distinguish between aversive and neutral cues during Pavlovian fear conditioning in behaving rats. Eur $J$ Neurosci 37: 498-507.

Moessnang C, Habel U, Schneider F, Siegel SJ (2012). The electrophysiological signature of motivational salience in mice and implications for schizophrenia. Neuropsychopharmacology 37: 2846-2854.

Moghaddam B (2002). Stress activation of glutamate neurotransmission in the prefrontal cortex: implications for dopamineassociated psychiatric disorders. Biol Psychiatry 51: 775-787.

Moghaddam B, Javitt D (2011). From revolution to evolution: the glutamate hypothesis of schizophrenia and its implication for treatment. Neuropsychopharmacology 37: 4-15.

Moghaddam B, Krystal JH (2012). Capturing the angel in 'angel dust': twenty years of translational neuroscience studies of NMDA receptor antagonists in animals and humans. Schizophr Bull 38: 942-949.

Murray GK, Corlett PR, Clark L, Pessiglione M, Blackwell AD, Honey $\mathrm{G}$ et al (2008). Substantia nigra/ventral tegmental reward prediction error disruption in psychosis. Mol Psychiatry 13: 267-276.

Ongür D, Prescot AP, McCarthy J, Cohen BM, Renshaw PF (2010). Elevated gamma-aminobutyric acid levels in chronic schizophrenia. Biol Psychiatry 68: 667-670.

Onn S-P, Wang X-B, Lin M, Grace AA (2006). Dopamine D1 and D4 receptor subtypes differentially modulate recurrent excitatory synapses in prefrontal cortical pyramidal neurons. Neuropsychopharmacology 31: 318-338.

Orliac F, Naveau M, Joliot M, Delcroix N, Razafimandimby A, Brazo P et al (2013). Links among resting-state default-mode network, salience network, and symptomatology in schizophrenia. Schizophr Res 148: 74-80.

Orona E, Gabriel M (1983). Multiple-unit activity of the prefrontal cortex and mediodorsal thalamic nucleus during acquisition of discriminative avoidance behavior in rabbits. Brain Res 263: 295-312.

O'Donnell P (2011). Adolescent onset of cortical disinhibition in schizophrenia: insights from animal models. Schizophr Bull 37: 484-492.

Paine TA, Slipp LE, Carlezon WA (2011). Schizophrenia-like attentional deficits following blockade of prefrontal cortex GABAA receptors. Neuropsychopharmacology 36: 1703-1713.

Palaniyappan L, Liddle PF (2012). Does the salience network play a cardinal role in psychosis? An emerging hypothesis of insular dysfunction. J Psychiatry Neurosci 37: 17-27.

Paxinos G, Watson C (2005). The rat brain in stereotaxic coordinates 5th edn. Academic Press: San Diego (CA).

Pehrson AL, Bondi CO, Totah NKB, Moghaddam B (2013). The influence of NMDA and GABA(A) receptors and glutamic acid decarboxylase (GAD) activity on attention. Psychopharmacology (Berl) 225: 31-39.

Pendyam S, Bravo-Rivera C, Burgos-Robles A, Sotres-Bayon F, Quirk GJ, Nair SS (2013). Fear signaling in the prelimbic- amygdala circuit: a computational modeling and recording study. J Neurophysiol 110: 844-861.

Quirk GJ, Russo GK, Barron JL, Lebron K (2000). The role of ventromedial prefrontal cortex in the recovery of extinguished fear. J Neurosci 20: 6225-6231.

Rao SG, Williams GV, Goldman-Rakic PS (2000). Destruction and creation of spatial tuning by disinhibition: GABA(A) blockade of prefrontal cortical neurons engaged by working memory. J Neurosci 20: 485-494.

Rascle C, Mazas O, Vaiva G, Tournant M, Raybois O, Goudemand M et al (2001). Clinical features of latent inhibition in schizophrenia. Schizophr Res 51: 149-161.

Roiser JP, Howes OD, Chaddock CA, Joyce EM, McGuire P (2013). Neural and behavioral correlates of aberrant salience in individuals at risk for psychosis. Schizophr Bull 39: 1328-1336.

Roiser JP, Stephan KE, Ouden HEM, den, Friston KJ, Joyce EM (2010). Adaptive and aberrant reward prediction signals in the human brain. Neuroimage 50: 657-664.

Rowland LM, Kontson K, West J, Edden Ra, Zhu H, Wijtenburg SA et al (2013). In vivo measurements of glutamate, GABA, and NAAG in schizophrenia. Schizophr Bull 39: 1096-1104.

Schiller D, Weiner I (2004). Lesions to the basolateral amygdala and the orbitofrontal cortex but not to the medial prefrontal cortex produce an abnormally persistent latent inhibition in rats. Neuroscience 128: 15-25.

Schneider PG, de Lores Arnaiz GR (2013). Ketamine prevents seizures and reverses changes in muscarinic receptor induced by bicuculline in rats. Neurochem Int 62: 258-264.

Sierra-Mercado D, Padilla-Coreano N, Quirk GJ (2011). Dissociable roles of prelimbic and infralimbic cortices, ventral hippocampus, and basolateral amygdala in the expression and extinction of conditioned fear. Neuropsychopharmacology 36: 529-538.

Simpson HB, Shungu DC, Bender J, Mao X, Xu X, Slifstein M et al (2012). Investigation of cortical glutamate-glutamine and $\gamma$ aminobutyric acid in obsessive-compulsive disorder by proton magnetic resonance spectroscopy. Neuropsychopharmacology 37: 2684-2692.

Sotres-Bayon F, Quirk GJ (2010). Prefrontal control of fear: more than just extinction. Curr Opin Neurobiol 20: 231-235.

Talk A, Stoll E, Gabriel M (2005). Cingulate cortical coding of contextdependent latent inhibition. Behav Neurosci 119: 1524-1532.

Taylor SF, Demeter E, Phan KL, Tso IF, Welsh RC (2013). Abnormal GABAergic Function and Negative Affect in Schizophrenia. Neuropsychopharmacology 39: 1000-1008.

Taylor SF, Phan KL, Britton JC, Liberzon I (2005). Neural response to emotional salience in schizophrenia. Neuropsychopharmacology 30: 984-995.

Vidal-Gonzalez I, Vidal-Gonzalez B, Rauch SL, Quirk GJ (2006). Microstimulation reveals opposing influences of prelimbic and infralimbic cortex on the expression of conditioned fear. Learn Mem 13: 728-733.

Volk DW, Lewis DA (2002). Impaired prefrontal inhibition in schizophrenia: relevance for cognitive dysfunction. Physiol Behav 77: 501-505.

Walter H, Heckers S, Kassubek J, Erk S, Frasch K, Abler B (2010). Further evidence for aberrant prefrontal salience coding in schizophrenia. Front Behav Neurosci 3: 1-9.

Weiner I (2003). The 'two-headed' latent inhibition model of schizophrenia: modeling positive and negative symptoms and their treatment. Psychopharmacology (Berl) 169: 257-297.

Weiner I, Arad M (2009). Using the pharmacology of latent inhibition to model domains of pathology in schizophrenia and their treatment. Behav Brain Res 204: 369-386.

Yuen EY, Yan Z (2009). Dopamine D4 receptors regulate AMPA receptor trafficking and glutamatergic transmission in GABAergic interneurons of prefrontal cortex. J Neurosci 29: 550-562. 